\title{
The Pigments of Sarcina flava: a new series of $\mathrm{C}_{50}$ Carotenoids
}

\author{
By D. THIRKELL AND R. H. C. STRANG \\ Department of Biochemistry, Bute Medical Buildings, St Andrews, Fife \\ AND J. R. CHAPMAN \\ Consultant Laboratory, A.E.I., Manchester \\ (Accepted for publication 29 April 1967) \\ SUMMARY
}

The carotenoids of Sarcina flava were examined; four main fractions were separated. These were hydrocarbons, mono- and di-hydroxylated compounds and a very polar fraction. Further separation of the hydrocarbon fraction showed the presence of nine compounds and of the very polar fraction which showed an all-trans form and three cis isomers. Data for these fractions is presented; at least two of the fractions are $\mathrm{C}_{50}$ carotenoids.

\section{INTRODUCTION}

After showing that Sarcina flava and S. lutea apparently synthesize identical pigments (Thirkell \& Strang, 1967), the following work was undertaken to examine the chemical nature of these carotenoids. Most carotenoids which have been reported to occur in bacteria have 40 carbon atoms, but recently a novel $\mathrm{C}_{50}$ carotenoid has been reported in Flavobacterium dehydrogenans (Jensen \& Weeks, 1966). The work reported here shows the existence of $\mathrm{C}_{50}$ carotenoids in another Gram-positive organism, S. flava.

\section{METHODS}

Bacteria. Large quantities of Sarcina flava (NTCC 7503) were grown on nutrient agar in large oblong aluminium dishes. The bacteria were harvested and stored at $-20^{\circ}$ until used.

Chemicals. All solvents used were Analar grade and were dried and redistilled before use. For spectrophotometric work, spectral quality solvents were used.

Pigment extraction and purification. Method (a) of Thirkell \& Strang (1967) was used and the total pigment obtained was first purified by the lipid precipitation technique of Blessin (1962). The ether of the resultant supernatant fluid was removed on a rotary evaporator under reduced pressure at $35^{\circ}$. The pigment was dissolved in methanol, made $10 \%(\mathrm{w} / \mathrm{v})$ with respect to $\mathrm{KOH}$ and saponified overnight at room temperature in the dark and in an atmosphere of nitrogen. The unsaponifiable material was extracted in the usual way.

Isolation of the individual pigment fractions. Preparative thin-layer chromatography on silica gel G (Merck) was used: $0.5 \mathrm{~mm}$. layers on $200 \times 200 \mathrm{~mm}$. plates were striploaded by using the mechanical applicator produced by Desaga (Camlab (Glass) Ltd.). The plates were developed in chloroform + methanol $(95+5, \mathrm{v} / \mathrm{v})$; this separated four main fractions. The solvent used in the previous work (Thirkell \& Strang, 1967) was not used in case the presence of a small concentration of acetic acid should induce the formation of isomers of certain fractions. 


\section{Tests used for characterization of fractions}

Acetylation (Kuhn \& Sorensen, 1938; Jensen, 1962). A sample of chromatographically pure material was taken to dryness on a rotary evaporator, and further dried under high vacuum for $30 \mathrm{~min}$. The fraction was dissolved in $\mathrm{I} \mathrm{ml}$. pyridine which had been dried over $\mathrm{NaOH}$ pellets and redistilled; $0.1-0.2 \mathrm{ml}$. acetic anhydride was added and the reaction allowed to proceed at room temperature in the dark under an atmosphere of nitrogen. Samples were withdrawn at regular intervals in order to follow the course of the reaction chromatographically over a period of $24 \mathrm{hr}$, by which time it was complete. The chromatography was done on $18.5 \mathrm{~cm}$. diameter kieselguhr-filled circular Schleicher \& Schüll chromatography papers (Jensen \& Jensen, 1959), using acetone and light petroleum (b.p. $60-80^{\circ}$ ) in various ratios as solvent. Thus the initial compound, the formation of intermediates during the reaction, and the formation of the final ester was followed. The ester formed was recovered into ether, and the ether thoroughly washed with water to remove pyridine. Spectrophotometric examination was then used to test that no degradation had occurred and to estimate the yield.

Test for tertiary hydroxyl groups after acetylation (S. L. Jensen, private communication). A sample of dry ester from acetylation was dissolved in $0.5 \mathrm{ml}$. dry pyridine and $0.2 \mathrm{ml}$. hexamethyl-disilane $+0.1 \mathrm{ml}$. trimethylchlorosilane added. The reaction proceeded at room temperature in an atmosphere of nitrogen. After I hr, carbon tetrachloride was added and the solution taken to dryness which removed the excess silane added. The product was dissolved in methanol and examined on kieselguhrfilled papers as before. The presence of a compound other than the substrate would be indicative of the formation of a silane from a tertiary hydroxyl group.

Reduction of esters with $\mathrm{LiAlH}_{4}$ (Goodwin, 1956). A small quantity of $\mathrm{LiAlH}_{4}$ was suspended in dry ether and the suspension filtered through glass wool to remove the larger particles. The filtered suspension was added to an ethereal solution of the ester in a separating funnel. After a short time, the reaction was terminated by adding wet ether. The products were removed, and excess $\mathrm{LiAlH}_{4}$ destroyed by adding water. Chromatography on kieselguhr-filled papers was again used to follow the reaction. The appearance of products other than the ester or original fraction would indicate that reducible groups were present.

Oxidation with nickel peroxide (S. L. Jensen, private communication). The fraction was dissolved in dry ether and nickel peroxide added in the ratio $5 \mathrm{mg}$. nickel peroxide to $\mathrm{I} \mathrm{mg}$. sample. The reaction occurs spontaneously at room temperature under nitrogen. Samples were taken at intervals, and the course of the reaction followed chromatographically as before. The test is specific for the presence of allylic hydroxyl groups.

Iodine isomerization (Zechmeister \& Polgár, 1943). To a solution of the fraction in acetone, a drop of iodine solution in hexane ( $10 \mu \mathrm{g} . / \mathrm{ml}$.) was added. The mixture was exposed to weak sunlight for $2-3 \mathrm{hr}$, samples were taken at intervals and the course of the reaction followed as before. When the pseudo-equilibrium was reached, the positions of the isomers were cut from the papers which were eluted with methanol, and the spectra and relative proportons determined.

Methylation of carboxyl group (Metcalfe \& Schmitz, 1961). A sample of the fraction under test was dried as for acetylation and dissolved in moisture-free methanol. 
$0.5 \mathrm{ml}$. methanolic boron trifluoride was then added and the reaction mixture refluxed for 2-3 min. The product was recovered into ether and examined chromatographically and spectrophotometrically.

Partition ratio (Petracek \& Zechmeister, 1956). A mixture of methanol +water $(95+5, v / v)$ and hexane was equilibrated and the sample was taken up in a known volume of one of them and transferred to a $10 \mathrm{ml}$. measuring cylinder. The sample was partitioned between equal volumes of both solvents and the two layers collected. The concentration of pigment in each layer was estimated spectrophotometrically.

Infrared spectroscopy. This was done with a Unicam SP $200 \mathrm{G}$ instrument, by using either liquid cells and solutions in carbon tetrachloride or carbon disulphide, or $\mathrm{KBr}$ discs (approximately $50 \mu \mathrm{g}$. sample/90 mg. dry $\mathrm{KBr}$ ).

Mass spectrometry. The sample was dissolved in the minimum volume of methylene dichloride and the solution spotted onto a ceramic direct insertion probe which was inserted through a vacuum lock into the ion chamber of an Associated Electrical Industries' MS 9 mass spectrometer. The sample was heated by contact with the walls of the ion chamber to achieve a reasonable rate of evaporation, and the mass spectrum scanned with the resolving power set at 1000 . The temperature of the ion chamber was about $250^{\circ}$. Masses of the peaks were measured very accurately at a resolving power of 12,000 .

\section{RESULTS}

The results of analysis of the four main fractions were as follows.

Fraction $I$ ( $5 \%$ of the total pigments). With chloroform + methanol $(95+5, \mathrm{v} / \mathrm{v})$ as solvent, this fraction ran with the solvent front. It was rechromatographed on silica-loaded papers (Whatman SG 8I) with light petroleum (b.p. 60-80 ) as solvent. This resolved the fraction into nine bands as documented in Table $\mathrm{I}$.

Table 1 . The nature of the carotene fractions resolved from fraction I by circular paper chromatography on silica-loaded papers (Whatman SG 8I) using light petroleum (b.p. $60-80^{\circ}$ ) as solvent

The partition ratio of each fraction between methanol + water $(95+5, v / v)$ and hexane, was $0 / 100$.

\begin{tabular}{|c|c|c|c|c|c|}
\hline Band & Type & $R_{F}$ value & Colour & $\lambda_{\max }$ in methanol & Comment \\
\hline $\mathrm{F}_{1}(a)$ & Fluorescent & 0.48 & Colourless & - & Early carotene precursor? \\
\hline (b) & Fluorescent & 0.40 & Colourless & - & Early carotene precursor? \\
\hline (c) & Absorbent & 0.28 & Yellow & $392,424,448$ & $?$ \\
\hline (d) & Fluorescent & 0.17 & Colourless & - & Very low concentration \\
\hline (e) & Absorbent & 0.12 & Yellow & $408,431,450$ & Unknown carotene \\
\hline (f) & Absorbent & $0 \cdot I_{I}$ & Yellow & $406,428,450$ & Isomer of F I $(e)$ \\
\hline$(g)^{*}$ & Absorbent & 0.07 & Yellow & $395,415,438$ & Unknown carotene \\
\hline$(h)^{*}$ & Absorbent & 0.06 & Yellow & $394,415,438$ & Isomer of F I $(g)$ \\
\hline (i)† & Absorbent & 0.03 & Yellow & $414,438,469$ & Sarcinene? \\
\hline
\end{tabular}

* Subfractions $(g)$ and $(h)$ did not separate completely but gave an elongated egg-shaped spot.

$\uparrow$ The $\lambda_{\text {ruax. }}$ of $F_{1}(i)$ are consistent with the presence of a chromophore containing nine conjugated double bonds. This is probably the parent hydrocarbon of the polar carotenoids in this bacterium.

Fraction 2 ( $8 \%$ of the total pigments). The results of the tests of this fraction are shown in Table 2. Some data obtained for this fraction and its derivatives from kieselguhr-filled circular chromatography papers are shown in Table 3. 
Fraction 3 (26\% of the total pigments). The results of tests on this fraction are shown in Table 4. Some data obtained for this fraction and its derivatives from kieselguhr-filled circular chromatography papers are shown in Table 5.

\section{Table 2. The results of chemical tests carried out on fraction 2 in an attempt to gain information as to its nature}

Test

Acetylation

Tertiary hydroxyl group

Reduction with $\mathrm{LiAlH}_{4}$

Oxidation with nickel peroxide

Iodine isomerization

Partition ratio

Infrared spectroscopy

Mass spectrometry
Result

Max. number of spots detected during reaction -2 (original

compound + an ester)

Final compound formedmonoester

Negative

Negative

One $\alpha-\beta$ unsaturated aldehyde formed

One isomer induced ( $\mathrm{Neo} U$ )

(20\% of original converted to isomer)

$27: 73$

No cis peaks; peak at 1050 $\mathrm{cm}^{-1}$

Parent ion at $\mathrm{m} / \mathrm{e}$ 686; mass measurement gave the empirical formula as $\mathrm{C}_{50} \mathrm{H}_{70} \mathrm{O}$
Comment

Single hydroxyl group present

No tertiary hydroxyl group present

No other reducible group present

One allylic hydroxyl group separated from the polyene chain

Polar compound, value consistent with monohydroxyl compound

All trans form; primary hydroxyl group

$\mathrm{C}_{50}$ carotenoid fraction

Table 3. Data obtained for fraction 2 and its derivatives from separation on kieselguhr-filled circular chromatography papers

The solvent used was acetone+light petroleum (b.p. $60-80^{\circ} ; 2+98, \mathrm{v} / \mathrm{v}$ ).

Sample

All trans fraction 2

Neo $\mathrm{U}$ isomer

Monoacetate
$\boldsymbol{R}_{\boldsymbol{F}}$ value

0.74

$0.6 \mathrm{I}$

0.95 $\lambda_{\max .}$ in methanol $(\mathrm{m} \mu)$

- $415,439,469$

$331,413,437,466$

$-, 415,439,469$

Fraction 4 (6r $\%$ of the total pigments). This polar fraction from the chloroform + methanol $(95+5, \mathrm{v} / \mathrm{v})$ separation was further separated on kieselguhr-filled circular chromatography papers into four fractions by using $40 \%$ acetone in light petroleum (b.p. $\left.60-80^{\circ}\right)$ as solvent. The four subfractions were: fraction $4(a)$, neo B isomer of fraction $4(c)(6.5 \%$ of fraction 4$)$; fraction $4(b)$, neo $\mathrm{A}$ isomer of fraction $4(c)$ ( $10.5 \%$ of fraction 4$)$; fraction $4(c)$, all trans compound $(53.0 \%$ of fraction 4$)$; fraction $4(d)$, neo $U$ isomer of fraction $4(c)(30.0 \%$ of fraction 4$)$. Fraction $4(c)$ was examined in more detail and the results are given in Table 6. Some data obtained for this fraction and its derivatives from kieselguhr-filled circular chromatography papers are given in Table 7 . 
Table 4. The results of chemical tests carried out on fraction 3 in an attempt to gain information as to its nature

Test

Acetylation

Tertiary hydroxyl group

Reduction with $\mathrm{LiAlH}_{4}$

Oxidation with nickel peroxide

Iodine isomerization

Partition ratio

Infrared spectroscopy

Mass spectrometry
Result

Max. number of spots detected during reaction-3 (original compound, mono- and di-ester).

Final compound formeddi-ester

Negative

Negative

One mono and one di $\alpha-\beta$ unsaturated aldehyde formed One isomer induced (Neo $\mathrm{U}$ ) (33\% original converted to the isomer)

71:29

No cis peaks; peak at $1050 \mathrm{~cm}^{-1}$

Parent ion at $\mathrm{m} / \mathrm{e} 702$; mass measurement gave the empirical formula as $\mathrm{C}_{50} \mathrm{H}_{70} \mathrm{O}_{2}$
Comment

Two hydroxyl groups present

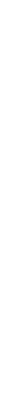

No tertiary hydroxyl group present

No other reducible group present

Two allylic hydroxyl groups separated from polyene chain

Polar compound, value consistent with the presence of two hydroxyl groups

All trans form; primary hydroxyl groups

$\mathrm{C}_{50}$ carotenoid fraction

Table 5. Data obtained for fraction 3 and its derivatives from separation on kieselguhr-filled circular chromatography papers

The solvent used was acetone + light petroleum (b.p. $60-80^{\circ} ; 10+90, \mathrm{v} / \mathrm{v}$ ).

Sample

All trans fraction 3

Neo $\mathrm{U}$ isomer

Monoacetate

Diacetate

$R_{P}$ value
0.50
0.42
0.60
0.99

Table 6. The results of chemical tests carried out on fraction $4(c)$ in an attempt to gain information as to its nature

Test

Result

Acetylation

Tertiary hydroxyl group

Reduction with $\mathrm{LiAlH}_{4}$ lodine isomerization

Partition ratio

Methylation of carboxyl group

Infrared spectroscopy
Max. number of spots detected during reaction-7. Final number of spots detected-I Negative

Not carried out on this fraction Three isomers were induced:

One Neo B isomer

One Neo A isomer

One Neo $\mathrm{U}$ isomer

100:0

Positive

No cis peak. No absolute conclusion could be reached from the traces as to the nature of the hydroxyl groups or as to the presence or absence of a carboxyl group $\lambda_{\text {max. }}$ in methanol $(\mathrm{m} \mu)$

$-, 415,439,469$

$331,412,436,466$

-, 415, 439, 469

$-, 415,439,469$ 
Table 7. Data obtained for fraction 4 and its derivatives from separation on kieselguhr-filled circular chromatography papers

The solvents used were *acetone+light petroleum (b.p. $\left.60-80^{\circ} ; 40+60, v / v\right)$ and †acetone + light petroleum (b.p. $60-80^{\circ} ; 10+90, \mathrm{v} / \mathrm{v}$ ).

\begin{tabular}{lcc}
\multicolumn{1}{c}{ Sample } & $R_{F}$ value & $\lambda_{\text {max. }}$ in methanol $(\mathrm{m} \mu)$ \\
Neo B isomer & $0.99^{*}$ & $33 \mathrm{I}, 4 \mathrm{I} 4,437,468$ \\
Neo A isomer & $0.83^{*}$ & $33 \mathrm{I}, 4 \mathrm{I} 4,438,468$ \\
All trans fraction $4(c)$ & $0.77^{*}$ & $-, 4 \mathrm{I} 6,439 \cdot 5,469 \cdot 5$ \\
Neo U isomer & $0.69^{*}$ & $33 \mathrm{I}, 4 \mathrm{I} 4,436,467$ \\
Triacetate & $0.67^{\circ}$ & $-, 4 \mathrm{I} 6,439 \cdot 5,469 \cdot 5$
\end{tabular}

\section{DISCUSSION}

This present work revealed the presence of a new series of $\mathrm{C}_{50}$ carotenoids in Sarcina flava (and presumably also in S. lutea; Thirkell \& Strang, 1967). We suggest that a $\mathrm{C}_{50}$ carotene (sarcinene ?; Chargaff \& Dieryck, 1932) would be the logical parent compound of the series. As fraction I (i) has the same chromophore as the other fractions, it is likely that this is the parent hydrocarbon in question. The addition of a further ten carbon atoms to the normal $\mathrm{C}_{40}$ skeleton may make the hydrocarbon relatively more polar as suggested by its low $R_{F}$ value. Examination of the other compounds in fraction $I$ by co-chromatography showed that the normal precursors of the $\mathrm{C}_{40}$ carotenoids, e.g. phytoene, phytofluene and neurosporene, were absent. This may suggest an alternative biosynthetic pathway for this series of compounds. On the basis of the evidence presented, it would seen that the parent hydrocarbon is first converted to a monohydroxyl compound (fraction 2), then to a dihydroxyl compound (fraction 3), and finally to a more polar compound (fraction 4).

Aged chloroform has a tendency to form a trace of $\mathrm{HCl}$ which could dehydrate allylic alcohols or induce isomerism in carotenoids. The chloroform used in this work was redistilled immediately before use, stored in the dark, and the chromatographic separations, which were reproducible, were done in an inert atmosphere. If cis isomers are present, appropriate $\lambda_{\max }$ are detected on a spectrophotometer. Further separation attempted on the fractions revealed the presence of cis isomers in fractions $I$ and 4. Two subfractions of fraction I appeared to be cis isomers, but the concentration of these fractions was very low. Fraction 4 was resolved into four subfractions on kieselguhr-filled chromatography papers using the solvent system as reported. Three of these subfractions were cis isomers and constituted $47 \%$ of fraction 4 . Using a different solvent system, Thirkell \& Strang (1967) also found cis isomers present in the pigments. of Sarcina flava, and whether or not these cis isomers are naturally occurring remains to be elucidated. It is extremely difficult to show that a cis isomer is naturally occurring. and not induced by a preparative procedure.

Under Dr S. L. Jensen's guidance, fraction 3 was compared with compound P 439 from Flavobacterium dehydrogenans (Jensen \& Weeks, 1966), the only previously reported $\mathrm{C}_{50}$ carotenoid. The two compounds gave identical results on chemical examination and neither the original compounds nor their derivatives could be separated by co-chromatography. Whereas compound $\mathrm{P} 439$ has an empirical formula of $\mathrm{C}_{50} \mathrm{H}_{72} \mathrm{O}_{2}$, the mono- and dihydroxyl fractions reported here have their most intense peak in mass spectral analysis corresponding to compounds with 70 hydrogen atoms. 
Evidence was also obtained which indicates the co-existence of both an $\mathrm{H}_{\mathbf{7 2}}$ and an $\mathrm{H}_{68}$ series. Other than mass spectral data, no evidence was found which suggested the presence of more than one carotenoid in fractions 2 and 3 .

The nature of fraction 4 is obscure. It forms a triple acetate, indicating three hydroxyl groups, but the $R_{F}$ value of this ester was still relatively polar and much more so than the esters produced from fractions 2 and 3. This polarity was not due to a tertiary hydroxyl group, since no silane derivative was produced. Some other polar group must be present; the methylation reaction indicated the possibility of a carboxyl group. This was not proved or disproved by infrared analysis. If the hydroxyl groups in fractions 2 and 3 are allylic and primary, hydroxylation of carbons 5 and 5 would be the most likely explanation. It is conceivable that one of these groups could be oxidized to carboxyl and that the three hydroxyl groups of fraction 4 are such that one primary hydroxyl group remains on carbon 5 or $5^{\prime}$, and that two others, presumably secondary hydroxyl groups, are substituted elsewhere on a ring.

It is not possible to arrive at the exact structure of any of the fractions but the following may be said. The work of Jensen \& Weeks (I966) has suggested the presence of two $\alpha$-ionone rings in compound $P_{439}$. Since $P_{439}$ gave identical chemical results and co-chromatographs with fraction 3 , it is reasonable to assume the presence of two $\alpha$-ionone rings in this series. Fractions 2 and 3 have primary hydroxyl groups (again similar to compound $\mathrm{P} 439$ ). The absorption spectra indicate that the main chromophore has nine conjugated double bonds and that the conjugated system does not extend into the rings. However, hydroxylation of methyl groups substituted on carbons 5 and $5^{\prime}$ of the rings may cause a spectral shift and thus definite proof of this proposed chromophore is still lacking. One would assume that the ten extra carbon atoms are derived from the inclusion of two extra isoprene units into the skeleton of these molecules, as compared with $\mathrm{C}_{40}$ carotenoids. This inclusion would be at some stage during biosynthesis and since many $\mathrm{C}_{40}$ carotenoids are mirror images about the central $\mathrm{C}_{15} 5^{-15^{\prime}}$ bond, it is most likely that one $\mathrm{C}_{5}$ unit is added on each side of this bond. The mass spectra of these compounds are not consistent with the $\mathrm{C}_{5}$ units being substituted either on the rings or on the central chain of carbon atoms. It may be that the central chain is elongated in some way so that the chromophore of nine conjugated double bonds is not altered.

The authors wish to thank Dr S. L. Jensen (Trondheim, Norway) for the help and encouragement given. One of the authors, R.H.C.S., was supported on an S.R.C. maintenance grant.

\section{REFERENCES}

BLEssin, C. W. (1962). Carotenoids of corn and sorghum. Cereal Chem. 39, 236.

Chargaff, E. \& Dieryck, J. (1932). Die Pigmente der S. lutea. Naturwissenschaften 20, 872.

GoodwIN, T. W. (1956). Studies in carotenogenesis. Biochem. J. 63, 48I.

Jensen, A. \& Jensen, S. L. (1959). Quantitative paper chromatography of lipids. Acta chem. scand. x3, 1863 .

JENSEN, S. L. (1962). The constitution of some bacterial carotenoids and their bearing on biosynthetic problems. Ph.D. Thesis, I Kommisjon Hos, Trondheim: F. Bruns Bokhandel.

Jensen, S. L. \& WeEks, O. B. (1966). A novel $\mathrm{C}_{50}$ carotenoid. Norwegian J. Chem., Mining and Metallurgy 26, 130.

KuHn, R. \& Sørensen, N. A. (1938). Uber Astaxanthin und Ovoverdin. Ber. dt. chem. Ges. 7r, 1879. MetCALFE, L. D. \& SCHMTT, A. A. (I96I). The rapid preparation of fatty acid esters for gas chromatographic analysis. Analyt. Chem. 33, 363 . 
PetraceK, F. J. \& ZeChMeISTER, L. (1956). Determination of partition coefficients of carotenoids as a tool in pigment analysis. Analyt. Chem. 28, 1484.

Thirkell, D. \& Strang, R. H. C. (1967). Analysis and comparison of the carotenoids of Sarcina lutea and S. flava. J. gen. Microbiol. 49, 53.

ZeCHMEISTER, L. \& Polgár, A. (I943). Cis-trans isomerism and spectrocharacteristics of carotenoids and some related compounds. J. Am. chem. Soc. 65, 1522. 